\title{
Use of progressive wave: a new multisource vibration technique to assist metal forming process
}

\author{
Armaghan Khan ${ }^{1, a}$, Thanh Hung Nguren ${ }^{1,2}$, Christophe Giraud-Audine ${ }^{2}$, \\ Gabriel Abba $^{1}$, Régis Bigot ${ }^{1}$ and Betty Lemaire-Semail ${ }^{2}$ \\ 1 LCFC, Arts et Métiers, 4 rue Augustin Fresnel, 57078 Metz, France \\ 2 L2EP, Université des Sciences et Technologies de Lille, 59655 Villeneuve d'Ascq, France
}

Received 17 June 2013, Accepted 15 September 2014

\begin{abstract}
Application of vibration in metal forming process has already shown numerous advantages in the past. It improves not only mechanical and surface properties of workpiece but the integration of vibration in metal forming process has also been helpful in the reduction of forming force required for the process. Majority of the work done before in this field is related to single vibration source vibration and its application in metal forming process. The aim of this work is to develop a mathematical model for multi vibration assisted forging process. The combination of multi mechanical vibrations generates a complicated movement of lower die without deforming it. This developed mathematical model proves that movement is basically in the form of progressive wave in the lower die thus resulting local surface movement. In reality this movement can be generated by the vibration given by multi piezoelectric actuators. Based on this mathematical model, simulations using finite element software Forge $2011^{\circledR}$ have been performed to observe the presence of progressive wave in the workpiece. The simulations' kinematics confirms the existence of progressive wave in the workpiece. Simulation results demonstrate the effect of progressive wave to reduce the forging force, reduction of friction on the lower surface of die and hence improvement the forging process. Based on the developed mathematical model and simulation results, design proposition for multi vibration assisted forging process has been presented in this work.
\end{abstract}

Key words: Mathematical modeling / forging process / piezoelectric actuators / progressive wave

\section{Introduction}

Due to the significance of metal forming processes in automotive, avionics and biomedical industries, rigorous efforts were made to improve these forming processes. Many techniques were developed for this purpose. The two traditional techniques to improve the forming process are the use of lubricants and preheating the material.

The major benefits of using lubricants are the reduction of forging load, tools' wear and improved surface finish (surface effect). The main limitation in using lubricants is the incompatibility with environment and its chemical reaction with tool/workpiece material. With the preheating method, the material's ductility increases while yield strength decreases (volume effect) but it affects important material properties such as surface structure, crystallographic structure and material strength as during heating there are voids, composition, and inclusion or precipitation formation.

\footnotetext{
${ }^{a}$ Corresponding author: Armaghan.Khan@ensam.eu
}

Application of vibration is the most recent and beneficial technique to improve the forming processes because it offers both surface and volume effects [1]. During the application of vibration in metal forming process, the contact between lower die and workpiece is periodic thus causing less friction between surfaces. Vibration also influences the movement of dislocation, producing plasticity which is the main aim of the forming processes. Moreover, it can prevent the undesirable effects related to two preceding techniques.

Until now, most of research in vibration assisted forming process concentrates on applying vibration in one direction using single vibration source. Vibration assisted forming processes can be divided into two domains depending upon the frequency application domains (ultrasonic and low frequency). Mostly researchers have applied ultrasonic vibration during different forming processes. For ultrasonic vibration assisted drawing process $[2-5]$, it was observed that drawing force required for the process has decreased in the presence of vibration. Reduction in friction between punch-workpiece 
(smooth surface) is observed along with reduction in wrinkling and cracking. Application of vibration in extrusion processes [6-10] has shown various advantages including reduction of extrusion force, friction and material flow stresses. Other important finding from these research works predicts increase in bursting pressure of pipe, tensile yield strength, higher crystallinity, larger crystal sizes and more perfect crystals. For vibration assisted upsetting processes [11-16], it was observed that there is reduction in mean upsetting/compression force, mean flow stresses, barreling, friction between die and workpiece with the integration of vibration in the process.

On the other hand, industrial application of ultrasonic vibration is limited by the power rating of ultrasonic generators [17] and also low frequencies are usually recommended, in order to avoid large reactive current in piezoelectric actuator. Vibration impact forging and forging with a superimposed vibration load (at 10-40 Hz frequency) gives a $50 \%$ increase in deformation and a similar reduction in specific pressure. At the same time, the tendency of the blank forged to assume a barrel-like shape is reduced, as a result of which both the stresses and strains are more uniformly distributed. During their research Ly et al. $[18,19]$ have worked on low vibration assisted forging process. Experimental setup incorporating piezoelectric actuator was developed to generate mechanical vibrations and it is placed under the lower die of Lloyd machine used for forging purpose. Norton-Hoff law was used to describe the viscoplastic behaviour of plasticine. Analytical model was developed to find the gain in forging force reduction and is compared with finite element simulations and experimental results. Khan et al. [20] use the same experimental setup and applied different types of waveforms during the upsetting process. It was observed that triangular vibration gave more forging force reduction as compared to sinusoidal vibration [21]. This gives a novel idea of using different waveforms in vibration assisted forming processes [22]. Some studies have been performed in the domain of orbital forging combined with translation $[23,24]$ and has shown tendency of obtaining surface and volume effects by changing frequency and amplitude of vibration.

Standing waves propagation in the tool used for microforming has been briefly discussed by Presz [1] that caused complicated usually $3 \mathrm{D}$ periodic deformation of tool resulting local surface movement so points of tool are moving on different ways. This research work concentrates on the propagation of progressive wave in the lower die due to the combination of vibration generated by multi vibration sources. This research work will consist of three main parts. The first part is the kinematic study and mathematical modeling of progressive wave by using multi vibration sources as it has long been mentioned that wave can be produced by mechanical vibration. The second part concentrates on finite element simulations performed in software Forge $2011^{\circledR}$ to see the impact of using progressive wave in the forging process and its effect on forging load's reduction. The third part of this work consists of design of mechanical system for multi vibration

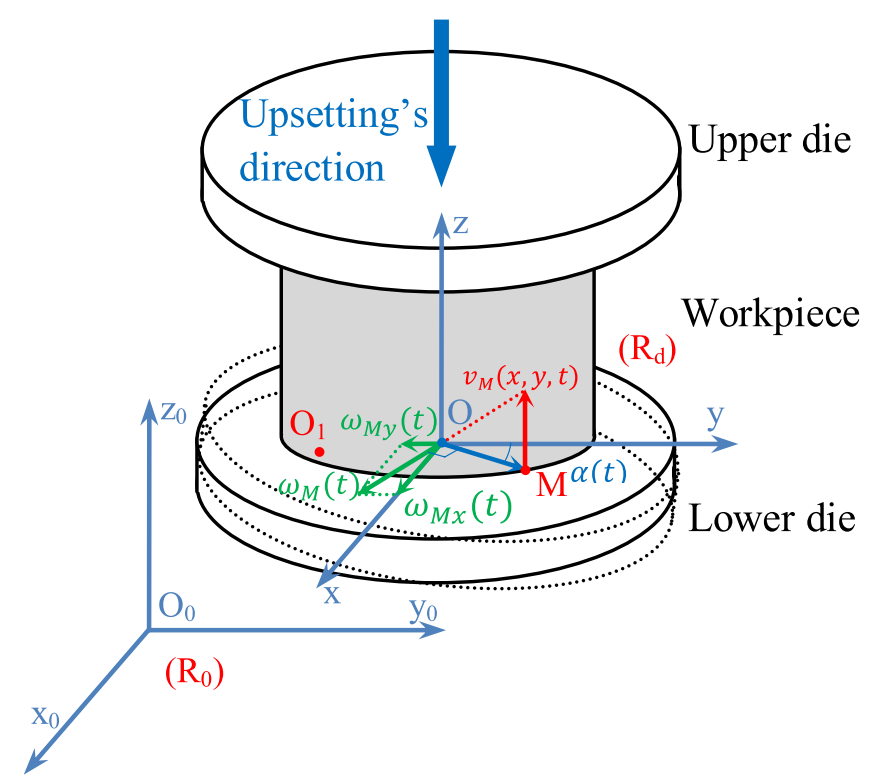

Fig. 1. Schematic view of the kinematics for the lower die in the forging process.

assisted forging and mathematical modeling to show the transmission of force and movement from piezoelectric actuators to lower die. Finally, conclusion and perspective of experimental verification will be outlined in the last section of this article.

\section{Kinematic model of the lower die and generation of progressive wave}

In order to perform forging of a cylindrical billet, lower die is the mechanical interface which will transmit the vibrations to billet. To generate complex vibration waveforms from the movement of multi piezoelectric actuators, the die must be guided by a mechanical system having a defined degree of freedom (dof). By limiting degree of freedom (dof), mechanical system facilitates controlled multiactuator system for a desired vibration. To establish a mathematical model of progressive wave on the lower die, the movement of two points in this die will be analyzed. Two cartesian frames are used in this study: $R_{0}$ is the one attached to the fixed base and $R_{\mathrm{d}}$ is the other attached to the lower die. In the $R_{\mathrm{d}}$ frame, a point $\mathrm{M}$ with coordinate $(x, y, 0)$ and $O_{1}$ with coordinate $\left(x_{1}, y_{1}, 0\right)$ are considered for kinematic study (Fig. 1). A cylindrical workpiece is put in contact with two die's surfaces and its symmetrical axis is on the $O_{z}$ axis.

If the movement of lower die is defined by two rotations (around $x$ and $y$ axes) and one translation (in $z$ direction), the rotation of the lower die expressed in the fixed base $R_{0}$ can be presented by the following matrix:

$$
R_{x} \cdot R_{y}=\left[\begin{array}{ccc}
1 & 0 & 0 \\
0 & 1 & -\theta_{x} \\
0 & \theta_{x} & 1
\end{array}\right]\left[\begin{array}{ccc}
1 & 0 & \theta_{y} \\
0 & 1 & 0 \\
-\theta_{y} & 0 & 1
\end{array}\right]=\left[\begin{array}{ccc}
1 & 0 & \theta_{y} \\
\theta_{x} \theta_{y} & 1 & -\theta_{x} \\
-\theta_{y} & \theta_{x} & 1
\end{array}\right]
$$


where $R_{x}, R_{y}$ are the matrices of rotation around $x$ and $y$ axes with the small angles of rotation $\theta_{x}$ and $\theta_{y}$. The position of points $M$ and $O$ can be defined in $R_{0}$ :

$$
\begin{aligned}
O_{0} M_{\left(R_{0}\right)} & =\left[\begin{array}{c}
x \\
y \\
-x \theta_{y}+y \theta_{x}+z
\end{array}\right] \\
O_{0} O_{1\left(R_{0}\right)} & =\left[\begin{array}{c}
x_{1} \\
y_{1} \\
-x_{1} \theta_{y}+y_{1} \theta_{x}+z
\end{array}\right]
\end{aligned}
$$

Now, if the movement at point $O_{1}$ is imposed as:

$$
-x_{1} \theta_{y}+y_{1} \theta_{x}+z=z_{a}(t)
$$

then the movement of point $M$ can be obtained as follows:

$$
O_{0} M_{\left(R_{0}\right)}=\left[\begin{array}{c}
x \\
y \\
-\left(x-x_{1}\right) \theta_{y}+\left(y-y_{1}\right) \theta_{x}+z_{a}(t)
\end{array}\right]
$$

If we assigned: $x-x_{1}=R \cos \psi$ and $y-y_{1}=R \sin \psi$, then we can have:

$$
z_{M}(t)=-R \cos \psi \theta_{y}+R \sin \psi \theta_{x}+z_{a}(t)
$$

We are therefore capable of choosing a sinusoidal progressive wave for the cylinder of radius $R$ and superposing a movement in $z$-direction defined by $z_{a}(t)$.

If we are only interested in the generation of progressive wave, then $\Delta z(t)=-R \cos \psi \theta_{y}+R \sin \psi \theta_{x}$ depends only to the radius $R$ of workpiece and the amplitudes of rotation angles. In the case of having an error in workpiece's centering in the lower die, a movement in z-direction is imposed on the workpiece in such way $z(t)=R_{1} \cos \psi_{1} \theta_{y}-R_{1} \sin \psi_{1} \theta_{x}$ with maximum amplitude $R_{1} \theta_{\text {max }}$.

On the other hand, if we want to achieve the sufficient amplitude at one point on the die to keep the workpiece on the die and to obtain the maximum velocity of deformation $V_{0}$, it is only important to consider the velocity at point $\mathrm{M}$ on the die. The lower die's radius determines the amplitudes of the movement around rotations' axes and the movement along $z$.

In this paper, only the progressive wave created by two rotations around two axes $O_{x}$ and $O_{y}$ will be studied. We consider the movement of a point $M$ at the lower surface of the workpiece in a distance $R$ from the center $O$. At time $t$, the position of point $M$ in the coordinate $O_{x y}$ is defined by an angle $\alpha$ between $O_{x}$ and $O M$.

The resultant angular velocity is $\vec{\omega}_{M}(t)=\vec{\omega}_{M, x}(t)+$ $\vec{\omega}_{M, y}(t)$ and is defined in Figure 1 and its magnitude is given by $\omega(t)=\sqrt{\omega_{x}^{2}+\omega_{y}^{2}}=\Omega_{0}$. The tangential velocity at point $\mathrm{M}$ is defined by $\vec{v}_{M}$ and is calculated as:

$$
\vec{v}_{M}(x, y, t)=\vec{\omega}_{M}(t) \times \overrightarrow{O M}
$$

In fact, point $M$ is on the radius $R$ of the matrix then $\overrightarrow{O M}=(R \cos \theta, R \sin \theta, 0)^{T}$, the tangential velocity at point $M$ is given by:

$$
\vec{v}_{M}(t)=\left|\begin{array}{cc}
\omega_{x} & \cos \theta \\
\omega_{y} & \sin \theta \\
0 & 0
\end{array}\right|=R \Omega_{0} \sin (\alpha t-\theta) \vec{z}
$$

The constant angular velocity vector $\vec{\omega}_{M}$ is projected on the two axes $O_{x}$ and $O_{y}$ and is given by:

$$
\begin{aligned}
& \omega_{M x}(t)=\Omega_{0} \cos \alpha \\
& \omega_{M y}(t)=\Omega_{0} \sin \alpha
\end{aligned}
$$

where $\Omega_{0}$ is the maximum amplitude of angular velocity and $\alpha=\omega t$, where $\omega$ is the angular velocity of vector $\vec{\omega}_{M}$ around $O_{z}$.

The angular displacements $\theta_{x}$ and $\theta_{y}$ can be calculated by the following equations:

$$
\begin{aligned}
& \theta_{x}(t)=\int_{0}^{t} \omega_{M x}(t)=\theta_{x_{0}}+\frac{\Omega_{0}}{\omega} \sin (\omega t) \\
& \theta_{y}(t)=\int_{0}^{t} \omega_{M y}(t)=\theta_{y_{0}}-\frac{\Omega_{0}}{\omega} \cos (\omega t)
\end{aligned}
$$

where $\theta_{x_{0}}$ and $\theta_{y_{0}}$ are the initial angles of rotation.

The upper die is supposed to move down at a constant velocity $V_{0}$. The instantaneous height reduction due to these dies' movements can be written as follows:

$h(t)=h_{0}-V_{0} t-R \theta(t)=h_{0}-V_{0} t-R \int_{0}^{t} \Omega_{0} \sin (\omega t+\phi) \mathrm{d} t$

where $h_{0}$ is the initial height of workpiece and $\theta(t)=$ $\int_{0}^{t} \Omega_{0} \sin (\omega t+\phi) \mathrm{d} t$ is the angle of rotation due to the angular velocity vector $\vec{\omega}_{M}$ at the point $M$ at the moment $t$ with the initial angle $\phi$.

Height reduction rate at point $M$ can be written:

$$
\dot{h}(t)=-V_{0}-R \Omega_{0} \sin (\omega t+\phi)
$$

From Equation (11), we can obtain $\dot{h}_{\max }=-V_{0}+R \Omega_{0}$. If $\dot{h}_{\max }>0$, the contact between the workpiece and dies is lost, therefore, the maximum value of $\dot{h}_{\max }$ is 0 . In that case, the maximum amplitude of the rotation depends on the defined velocity $V_{0}$ and the radius of the workpiece:

$$
\Omega_{0 \max }=\frac{V_{0}}{R}
$$

In combination with this progressive wave movement, a low frequency below $100 \mathrm{~Hz}$ can also be superimposed on the point $O$ of the lower die to investigate the effect of uniaxial vibration. The system must therefore be capable of performing two rotations around two axes $O x$ and $O y$ and one translation along the axis $O z$. A kinematic diagram of the mechanical system with required 3 dof is shown in Figure 2 where the two corresponding rotations occur at the pivots and translation is materialized by sliding connection. However, the use of elastic guiding in place of the conventional guiding must be considered to prevent the 


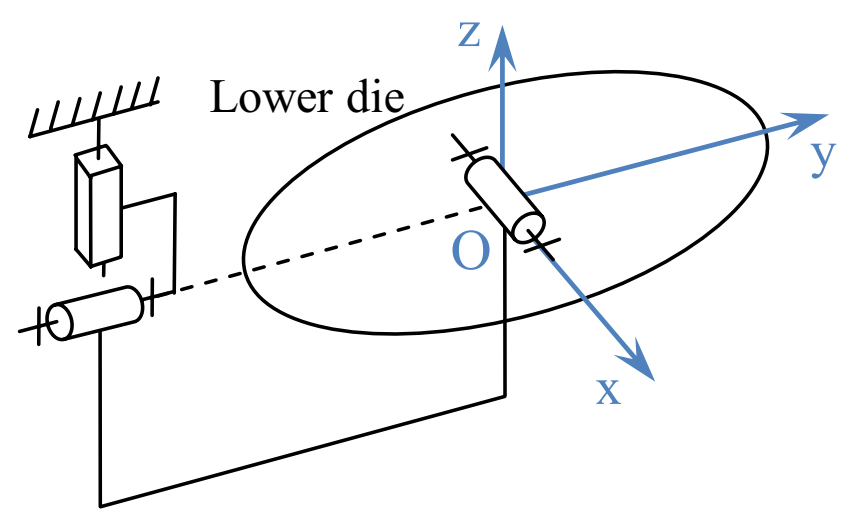

Fig. 2. Kinematic scheme of mechanical system.

mechanical backlash which is incompatible with the very small required displacements.

The piezoelectric actuators, which are widely used for this kind of application [18], are promising sources of vibrations to meet these requirements. However, a methodology for designing and controlling mechanical system integrating multi piezoelectric actuators must be studied further to realize the proposed kinematic movement.

\section{Design of the mechanical system for multi vibration assisted forging process}

\subsection{Design of the mechanical system}

To benefit from the high rigidity and strength of multi layer piezoelectric actuators, the actuators must be placed vertically to oppose the forging force. With the vertical position of the actuators, a configuration with at least three actuators arranged equidistantly from the center tray and a $120^{\circ}$ angle is given in Figure 3 . This arrangement allows, by controlling the elongation of the actuators, to define the inclination around $O x, O y$ plane of the matrix and its elevation along $O z$. In addition, it allows to oppose the tilting of the case of the eccentric load. If the plot is properly centered, this configuration provides a balanced distribution of forging force on 3 actuators.

To achieve the equivalent movement of the lower die as a progressive wave, it is necessary that the movement of the actuators is synchronized. The instantaneous speed of each actuator is determined by the instantaneous rotation vector of the lower die given by Equation (8). Figure 4 can be used to determine the expression for the vertical velocity for each actuator.

As can be seen in Figure 4, the terms of the vertical speed of the actuators are provided by:

$$
\begin{aligned}
& \vec{v}_{1}=R_{1} \Omega_{0} \sin \left(\frac{\pi}{2}-\alpha(t)\right) \vec{z} \\
& \vec{v}_{2}=R_{1} \Omega_{0} \sin \left(\frac{\pi}{2}-\frac{2 \pi}{3}-\alpha(t)\right) \vec{z} \\
& \vec{v}_{3}=R_{1} \Omega_{0} \sin \left(\frac{\pi}{2}-\frac{4 \pi}{3}-\alpha(t)\right) \vec{z}
\end{aligned}
$$

These relationships also show the advantage of this configuration which resembles like three phase voltage where the speeds of the actuators are placed to form a three phase system.

\subsection{Transmission of forces and movements to lower die}

In this section, transmission of forces and movements applied by piezoelectric actuator on the lower die will be discussed. The mechanical system shown in Figure 5 takes into account the different established steps involved in the transmission of the movement from piezoelectric actuators to the center of the lower die. These steps consist of the force and velocity transmission through contacts' support, different sphere-plane contacts and secondary plate.

The purpose is to study the kinematics of lower die in real system. Schematic of resultant forces applied on the lower die during the forging process is presented in Figure 6. In general, movement of the mechanical system consists of an elastic guidance system, secondary plate and the lower die.

External forces applied to point $O$, the center of lower die are Flopin and $F_{1}, F_{2}, F_{3}$ as shown in Figure 6 . In this study, the influence of the frictional contact and the change in orientation of the contact due to the rotations of the plate are neglected. Therefore, these forces are assumed vertical and moments are neglected at these points. These forces come from the transmission of forces generated by 3 piezoelectric actuators $F_{p, 1}, F_{p, 2}, F_{p, 3}$ and forging $F_{f}$ (Fig. 6). Taking in consideration, the orders of size and speed of the lower die $(90 \mathrm{~mm}$ diameter and maximum speed of $6 \mathrm{~mm} . \mathrm{s}^{-1}$ ), the forces generated by actuators $F_{p}, I$ and forging $F_{f}$ (of the order of several $k N$ ) are dominant over the effects of gravity, damping and centrifugal forces. In this study, it was considered that if the contact carriers have a mass of about $1 \mathrm{~kg}$, the influence of inertia is of the order of hundreds of Newton which means that the forces $F_{1}, F_{2}, F_{3}$ have orders of magnitudes similar to those of the forces generated by the actuators.

Force and moments $F_{z}, M_{x}, M_{y}$ due to the force of contact concentrated at the center of the plate are obtained by reduction of the torsor of these three forces applied by the piezoelectric actuators at contact point $O$. Points of application of force of contact on the secondary plate are shown in Figure 7. Tangential displacements of the points of contact in the secondary plate caused by the rotation of the plate can be neglected. Change in the orientation of the contact force is also assumed to be negligible. In addition, the effect of the friction is neglected and we shall only considered the forces and velocities of the contacts in the $z$ direction, which are dominant as compared to those in the plane perpendicular to the $z$ axis. $\overrightarrow{v_{1}}, \overrightarrow{v_{2}}$ and $\overrightarrow{v_{3}}$ are the three instantaneous velocity vectors of points of contact $I i$ in $z$ direction which belongs to actuator axis. In order to find a relationship between the velocity of the point $O$ in $O_{z}$ and three velocities $\overrightarrow{v_{1}}$, $\overrightarrow{v_{2}}$ and $\overrightarrow{v_{3}}$, we consider the influence of each velocity vector $\overrightarrow{v_{1}}, \overrightarrow{v_{2}}$ and $\overrightarrow{v_{3}}$ at point $O$. We recall that $\overrightarrow{\omega_{1}}, \overrightarrow{\omega_{2}}$ et $\overrightarrow{\omega_{3}}$ 

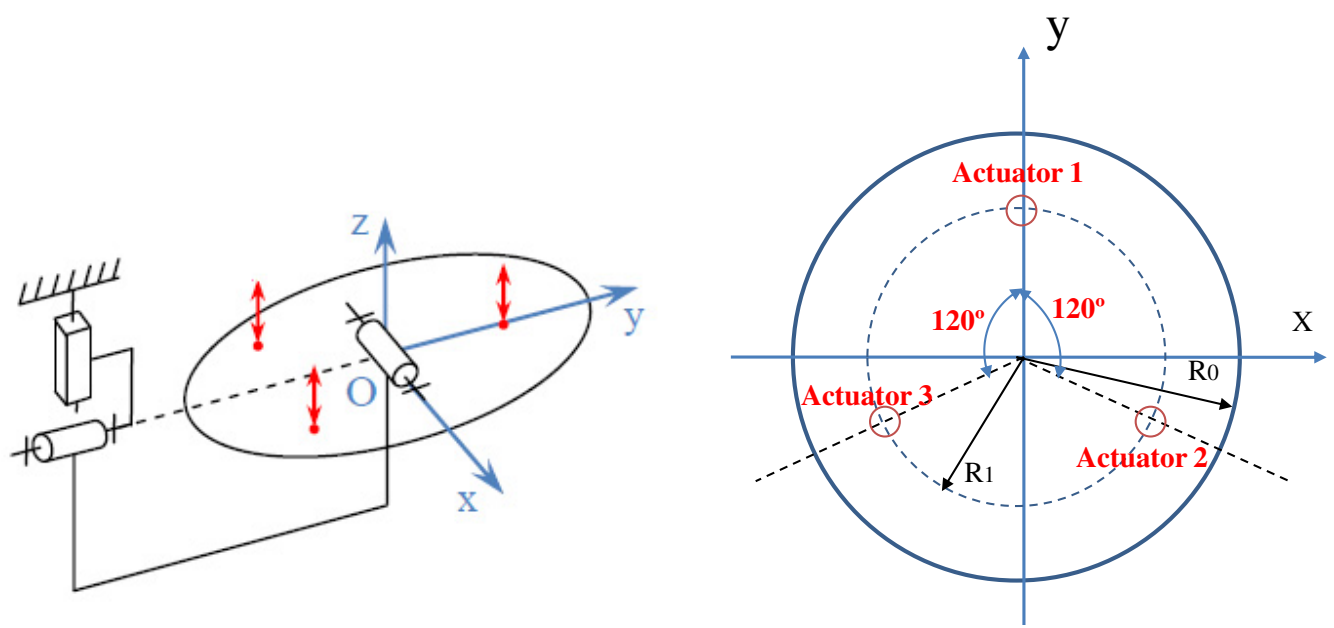

Fig. 3. Configuration of 3 piezo actuators mechanical system.

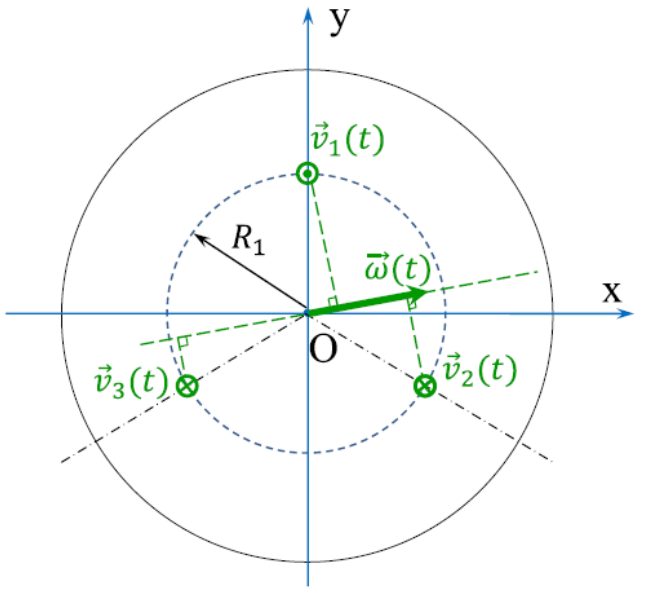

Fig. 4. Velocity of actuators defined by vector of rotation of lower die.

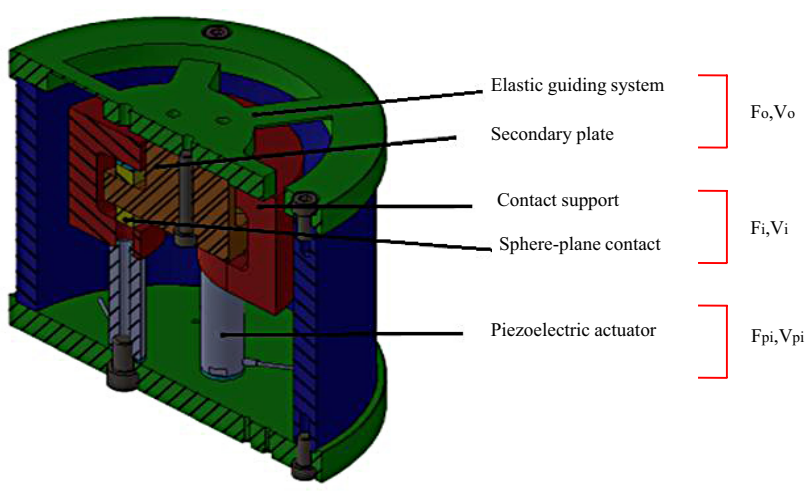

Fig. 5. Design of mechanical system consisting of three piezoelectric actuators.

are velocity vectors of instantaneous rotation due to each velocity vector of displacement $\overrightarrow{v_{1}}, \overrightarrow{v_{2}}$ et $\overrightarrow{v_{3}}$ respectively (Fig. 8). When one of the actuator is energized, the other remains at rest, the instantaneous axis of rotation passes

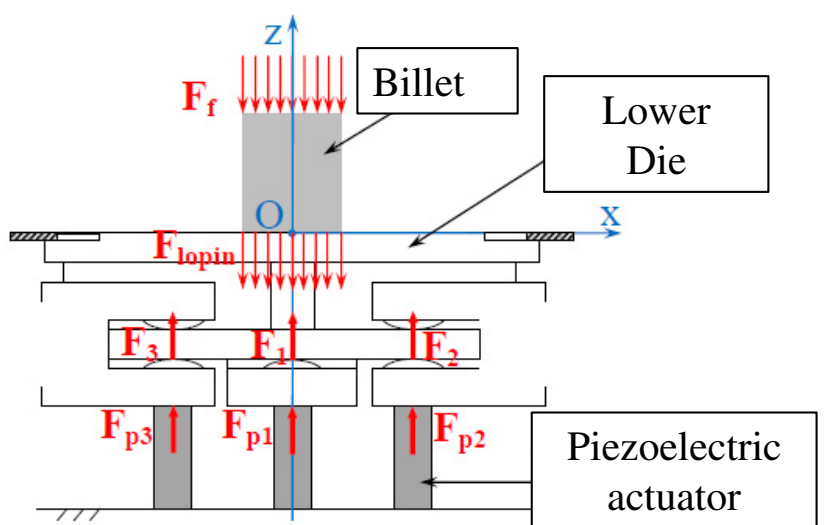

Fig. 6. Schematic of the forces applied on lower die during forging process.
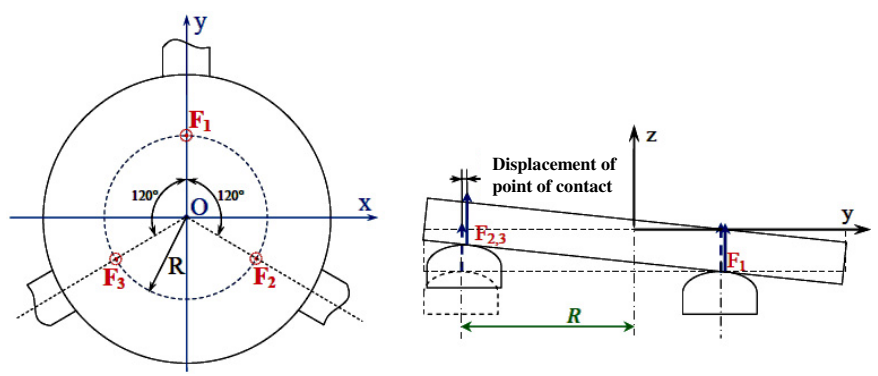

Fig. 7. Schematic of the forces applied on secondary plate.

through the contact points corresponding to them. The rotation vector (angular velocity) $\overrightarrow{\omega_{i}}$ is defined by:

$$
\overrightarrow{v_{i}}=\overrightarrow{I_{i} P_{i}} \times \overrightarrow{\omega_{i}}
$$

The kinematic torsor at point $O$ caused by the velocity $\overrightarrow{v_{i}}$ can be written as:

$$
\left\{\begin{array}{c}
\overrightarrow{\omega_{i}} \\
\overrightarrow{v_{i} O}
\end{array}\right\}=\left\{\begin{array}{c}
\overrightarrow{\omega_{i}} \\
\overrightarrow{O P_{i}} \times \overrightarrow{\omega_{i}}
\end{array}\right\}
$$


For small displacements, the kinematic torsor at point $O$ is calculated by:

$$
\left\{\begin{array}{l}
\overrightarrow{\omega_{O}} \\
\overrightarrow{v_{O}}
\end{array}\right\}=\sum\left\{\begin{array}{c}
\overrightarrow{\omega_{i}} \\
\overrightarrow{v_{i} / O}
\end{array}\right\}=\left\{\begin{array}{c}
\sum \overrightarrow{\omega_{i}} \\
\sum \overrightarrow{O P_{i}} \times \overrightarrow{\omega_{i}}
\end{array}\right\}
$$

The projection of Equation (16) at three axes $O_{x}, O_{y}, O_{z}$ gives:

$$
\begin{aligned}
& \left\{\begin{array}{l}
v_{z}=\frac{1}{3}\left(v_{1}+v_{2}+v_{3}\right) \\
\omega_{x}=\omega_{1}-\omega_{2} \sin \frac{\pi}{6}-\omega_{3} \sin \frac{\pi}{6} \\
\omega_{y}=\omega_{3} \cos \frac{\pi}{6}-\omega_{2} \cos \frac{\pi}{6}
\end{array}\right. \\
& \Rightarrow\left\{\begin{array}{l}
v_{z}=\frac{1}{3} v_{1}+\frac{1}{3} v_{2}+\frac{1}{3} v_{3} \\
\omega_{x}=\left(\frac{2}{3 R}\right) v_{1}-\left(\frac{2}{3 R} \sin \frac{\pi}{6}\right) v_{2}-\left(\frac{2}{3 R} \sin \frac{\pi}{6}\right) v_{3} \\
\omega_{y}=\left(\frac{2}{3 R} \cos \frac{\pi}{6}\right) v_{3}-\left(\frac{2}{3 R} \cos \frac{\pi}{6}\right) v_{2}
\end{array}\right. \\
& \Rightarrow\left\{\begin{array}{l}
v_{1}=v_{z}+R \omega_{x} \\
v_{2}=v_{z}-\frac{R}{2} \omega_{x}-\frac{\sqrt{3} R}{2} \omega_{y} \\
v_{3}=v_{z}-\frac{R}{2} \omega_{x}+\frac{\sqrt{3} R}{2} \omega_{y}
\end{array}\right. \\
& {\left[\begin{array}{l}
v_{1} \\
v_{2} \\
v_{3}
\end{array}\right]=\left[\begin{array}{ccccc}
0 & 0 & 1 & R & 0 \\
0 & 0 & 1 & -\frac{R}{2}-\frac{R \sqrt{3}}{2} & 0 \\
0 & 0 & 1 & -\frac{R}{2} \frac{R \sqrt{3} R}{2} & 0
\end{array}\right]\left[\begin{array}{l}
v_{x} \\
v_{y} \\
v_{z} \\
\omega_{x} \\
\omega_{y} \\
\omega_{z}
\end{array}\right]=\mathbf{A} \mathbf{v}_{\text {contact }}}
\end{aligned}
$$

The relationship between the forces applied at contacts and the resulting force at center $O$ can be found by concentrating the force torsors at the center of plate. The force of contacts considered in this case is assumed vertical as assumptions before. Note that $\overrightarrow{M_{1}}, \overrightarrow{M_{2}}$ and $\overrightarrow{M_{3}}$ are the instantaneous moments caused by each vertical component of force of contact $\overrightarrow{F_{1}}, \overrightarrow{F_{2}}$ and $\overrightarrow{F_{3}}$ as shown in Figure 9 .

The static torsor at point $O$ can be calculated as:

$$
\begin{gathered}
\left\{\begin{array}{l}
\overrightarrow{F_{O}} \\
\overrightarrow{M_{O}}
\end{array}\right\}=\sum\left\{\begin{array}{l}
\vec{F}_{i} \\
\overrightarrow{M_{i / O}}
\end{array}\right\}=\left\{\begin{array}{c}
\sum \overrightarrow{F_{i}} \\
\sum \overrightarrow{O I_{i}} \times \overrightarrow{F_{i}}
\end{array}\right\} \\
\left\{\begin{array}{l}
F_{z}=F_{1}+F_{2}+F_{3} \\
M_{x}=R F_{1}-F_{2} R \sin \frac{\pi}{6}-F_{3} R \sin \frac{\pi}{6} \\
M_{y}=F_{3} R \cos \frac{\pi}{6}-F_{2} R \cos \frac{\pi}{6}
\end{array}\right.
\end{gathered}
$$

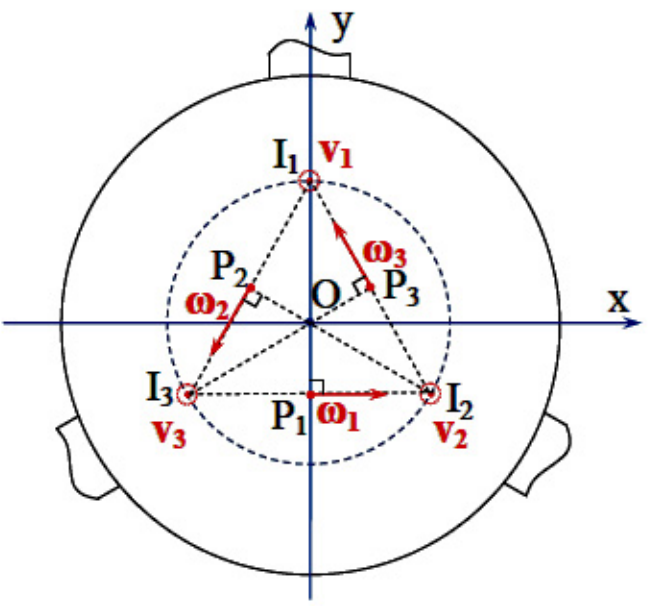

Fig. 8. Schematic of the kinematic tensor.

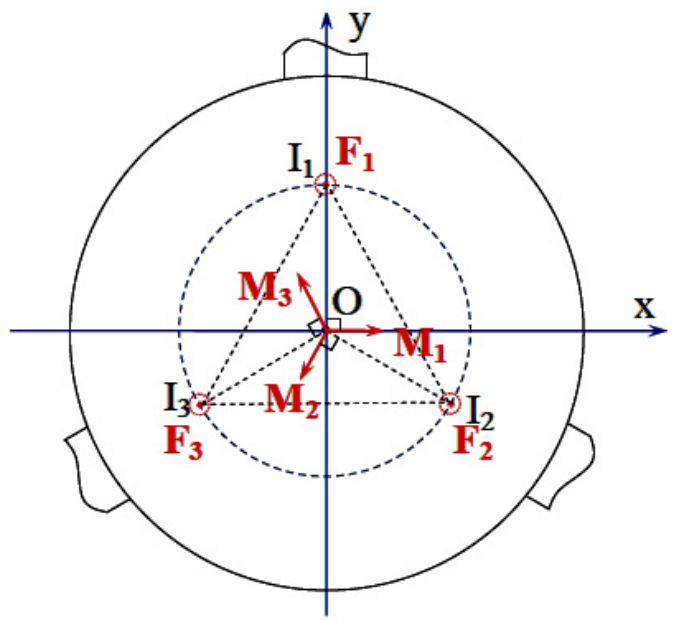

Fig. 9. Schematic of the static tensor.

So, the resultant vector of force of contact $\mathbf{F}_{\text {contact }}$ can be written in matrix form as:

$$
\mathbf{F}_{\text {contact }}=\left[\begin{array}{c}
F_{x} \\
F_{y} \\
F_{z} \\
M_{x} \\
M_{y} \\
M_{z}
\end{array}\right]=\left[\begin{array}{ccc}
0 & 0 & 0 \\
0 & 0 & 0 \\
1 & 1 & 1 \\
R & -\frac{R}{2} & -\frac{R}{2} \\
0 & -\frac{R \sqrt{3}}{2} & \frac{R \sqrt{3}}{2} \\
0 & 0 & 0
\end{array}\right]\left[\begin{array}{c}
F_{1} \\
F_{2} \\
F_{3}
\end{array}\right]=\mathbf{B}\left[\begin{array}{c}
F_{1} \\
F_{2} \\
F_{3}
\end{array}\right]
$$

It can be noted that:

$$
\mathbf{B}^{\top}=\mathbf{A}
$$

To establish the energetic macroscopic representation (EMR), it is necessary to study the properties of this mechanical coupling with energy conservation point of view. The power developed by the contact forces and applied to the plate is calculated by:

$$
P=\mathbf{F}_{\text {contact }}^{\top} \mathbf{v}_{\text {contact }}
$$




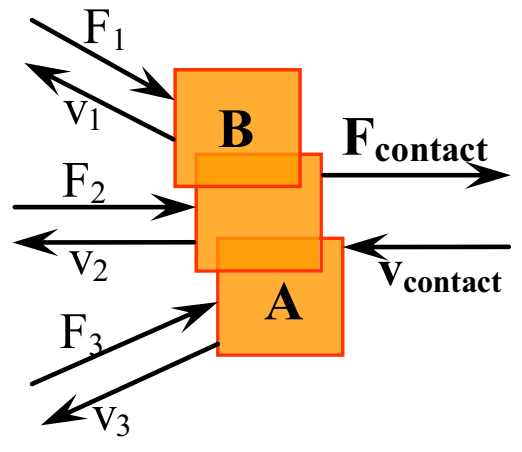

Fig. 10. EMR of mechanical coupling.

Using the relationship (19), it becomes:

$$
P=(\mathbf{B F})^{\top} \mathbf{v}_{\text {contact }}=\mathbf{F}^{\top} \mathbf{B}^{\top} \mathbf{v}_{\text {contact }}
$$

which, taken in account the property (20), can be written as:

$$
P=\mathbf{F}^{\top} \mathbf{A} \mathbf{v}_{\text {contact }}
$$

Finally, from the kinematic relationship (17):

$$
P=\mathbf{F}^{\top} \mathbf{v}
$$

It can be seen from this calculation that the model satisfies the conservation of power which allows to check the consistency of the approximations made and allows it to associate a macroscopic energy representation as proposed in Figure 10.

\section{Finite element (FEM) simulation for forging process with multisource vibrations}

Finite element simulations have been performed in viscoplastic domain for forging processes with and without vibrations in FORGE2011 ${ }^{\circledR}$. FEM simulations with single vibration has earlier been performed in viscoplastic domain using Norton Hoff law $[18,19]$ and has been verified analytically and experimentally. Since this paper also focuses on a viscoplastic behavior, the material behavior will be expressed by Norton-Hoff constitutive law (extracted from Hansel and Spittel rheology law) [21], according to which:

$$
\sigma_{0}=k \varepsilon^{n} \dot{\varepsilon}^{m}
$$

From Equation (23), it can be seen that the flow stress $\sigma_{0}$ depends on the equivalent deformation $\varepsilon$, the equivalent deformation rate $\dot{\varepsilon}$ along with the coefficients of material (the consistency $k$, the hardening coefficient $n$ and the sensitivity to strain rate $m$ ).

Because this study is about cold forging process, the temperature terms are not included in this model. Friction is taken into account by using a Coulomb limited Tresca model as it was taken in case of single vibration

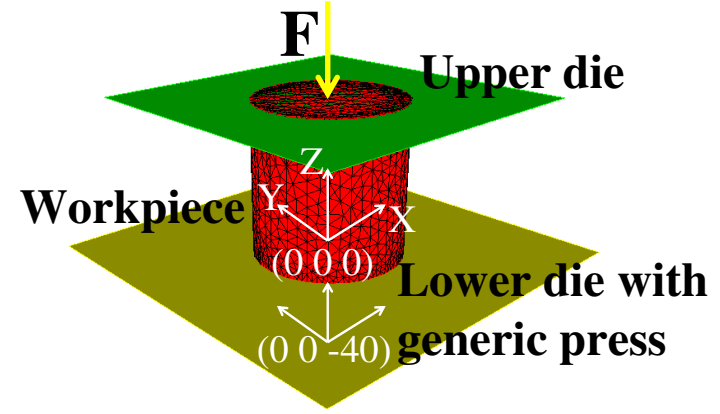

Fig. 11. FORGE2011 ${ }^{\circledR}$ model for FEM simulations.

(source) [21] to facilitate the comparison. All the friction and material parameters are kept same as before [21].

The input data for this simulation are the two angular velocities generated by Equations (9) and transferred to software FORGE2011 ${ }^{\circledR}$ with the help of generic press for further use during the forging process. Figure 11 presents the FORGE model, showing the three main parts of the forging process (upper die, workpiece and lower die).

In these simulations, aluminum was used as workpiece material to perform forging process. Other materials can also be used, as material coefficients have to be changed accordingly in the workpiece material file. Table 1 summarizes the different parameters of model and process used in these simulations.

The simulation starts at time $t=0$, then the initial angular displacements $\theta_{x 0}, \theta_{y 0}$ are calculated from Equation (9) as follows:

$$
\theta_{x}(0)=0 ; \quad \theta_{y}(0)=0 ;
$$$$
\Rightarrow \theta_{x 0}=0 ; \quad \theta_{y 0}=\frac{\Omega_{0}}{2 \pi f}=\frac{V_{0}}{2 \pi f R}
$$$$
=-0.00239 \mathrm{rad}=-0.137^{\circ}
$$

Presence of rotation in the lower die can be found out by measuring displacement for small period of time. Similarly, the presence of rotation on the lower surface of workpiece can be measured with the help of a-priori sensors during the simulation. This option is available in the FEM software FORGE2011 ${ }^{\circledR}$. These sensors can also be used to find out other important data measurement such as total strain, strain rate, displacement and velocities in $x, y$ and $z$ directions at any time during the simulation.

Once the presence of rotation at the upper surface of lower die and in the lower surface of workpiece has been verified, the effect of the multi vibration on the forging process can be observed. This can be done by comparing the forging load required for the processes with and without multi vibration.

This comparison has been presented in Figure 12. Figure $12 \mathrm{a}$ presents load required for the forging processes with and without vibration. It shows that there is forging load reduction when multi vibrations have been applied.

Normalized load reduction can be found out by normalizing the forging load over the time. Normalized load 
A. Khan et al.: Mechanics \& Industry 16, 108 (2015)

Table 1. FEM model parameters used for forging process simulations.

\begin{tabular}{cc}
\hline \multicolumn{2}{c}{ Parameters and values } \\
\hline Die (Steel) & Non deformable \\
Workpiece material coefficient (Aluminum) & $k=161.9 ; n=0.2 ; m=0.1$ \\
Friction coefficients & $\mu=0.05, m=0.8$ \\
Workpiece geometry & $\mathrm{R}=20 \mathrm{~mm} ; h_{0}=40 \mathrm{~mm}$ \\
Workpiece final height & $h_{f}=15 \mathrm{~mm}$ \\
Upper die velocity & $V_{0}=-3 \mathrm{~mm} . \mathrm{s}^{-1}$ \\
Progressive wave frequency & $f=10 \mathrm{~Hz}$ \\
Rotation velocity & $\Omega_{0}=\Omega_{0 \text { max }}=0.15 \mathrm{rad} . \mathrm{s}^{-1}$ \\
\hline
\end{tabular}

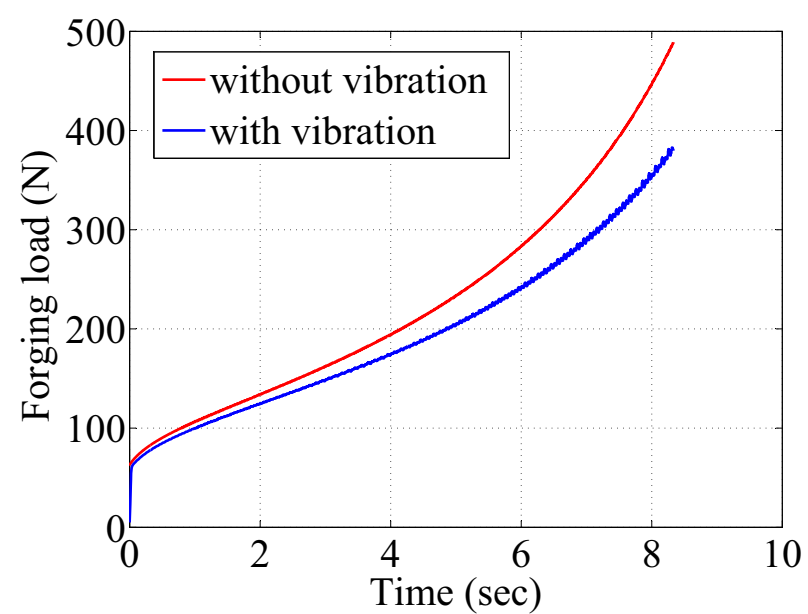

(a)

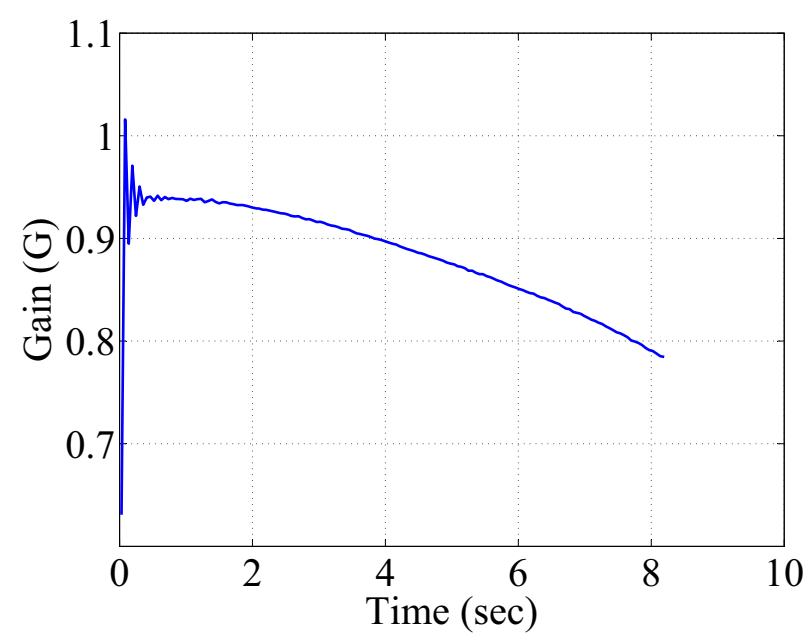

(b)

Fig. 12. (a) Load versus time for forging processes with and without multi vibration. (b) Gain in forging load reduction: $f=10 \mathrm{~Hz}$ and $\Omega_{0 \max }=0.15 \mathrm{rad} . \mathrm{s}^{-1}$.

reduction or gain in forging load reduction (ratio of forging load with vibration and without vibration $G=\frac{F_{v}}{F_{0}}$ is presented in Figure 12b. Forging load reduction of $21.5 \%$ has been obtained at the end of the process. It can be seen that initially gain in forging load reduction is small but with time, this gain has increased as plastic deformation increases. This phenomenon shows the advantage of using progressive wave in the forging process.

In Figure 13, the velocity field has been plotted for two time instants during the time period $T=0.1 \mathrm{~s}(t=$ $2-2.1 \mathrm{~s})$. It can be clearly seen that there is change in the velocity vector's direction from the right side of workpiece in Figure $4 \mathrm{a}$ to the left side of workpiece in Figure $4 \mathrm{~b}$ during a half period of the progressive wave. In one of two cases, the direction of velocity field direction is opposing the slip direction and in second case it is supporting slip. This explains the reduction of the stress in the material and the reduction of the friction on the lower die.

\section{Conclusions and future work}

In this work, presence of progressive wave is predicted with the help of two rotations generated by multiple vibration source during the forging process. The kinematic study has been presented and applied during the forging process simulation performed in the Forge $2011^{\circledR}$. Simulation's results confirm the gain in forging load reduction. Idea of applying multi-vibration sources to generate progressive wave during the forging process has been discussed for the first time and an experimental approach to employ it is in process. For this purpose, mechanical design of an experimental setup has been proposed which details the transmission of force and movement from piezoelectric actuator to lower die of the forging or compression machine. Progressive wave can be generated with the help of two rotations but in reality we need three piezoelectric actuators to produce rotation in two directions. Decision of obtaining two rotations and one translation can be authorized with the help of programming through micro controller. In addition, vertical movement (translation) in $z$ direction can give additional advantage in forging load reduction.

In the future work, experiment needs to be performed based on the mechanical design developed for this purpose. A mechanical system must be designed with the strict conditions to support the forging load (up to $12 \mathrm{kN}$ ) and generate the displacements (in order of $\mu \mathrm{m}$ ) to perform a proposed progressive wave of movement in the lower die. 

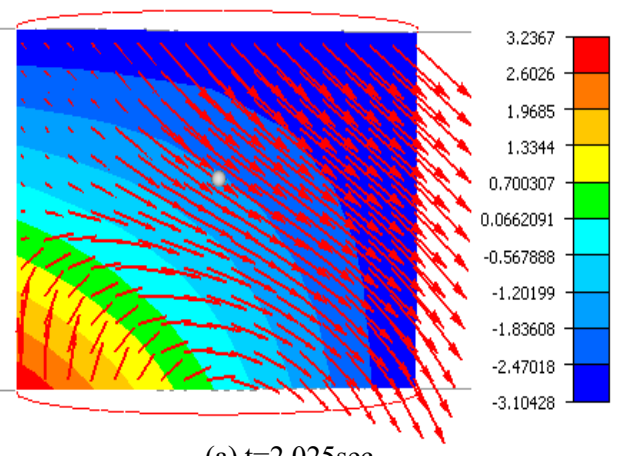

(a) $\mathrm{t}=2.025 \mathrm{sec}$
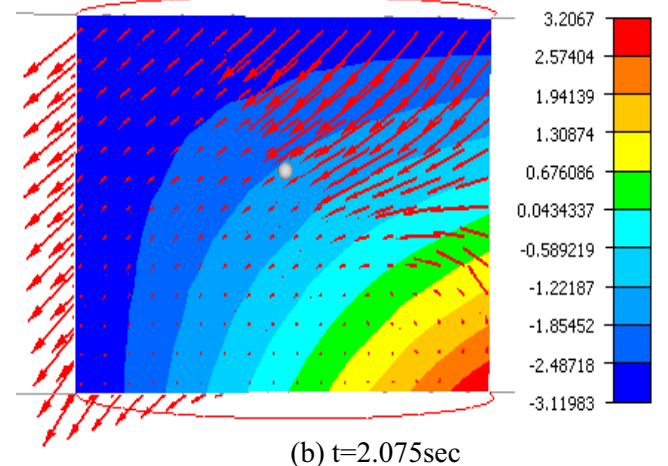

Fig. 13. Velocity field of workpiece (a) $t=\frac{T}{4}$, (b) $t=\frac{3 T}{4}$.

\section{References}

[1] W. Presz, Flexible Manufacturing System For Vibration Assisted Microforming, J. Achiev. Mater. Manuf. Eng. 21 (2007) 61-64

[2] M. Hayashi, M. Jin, S. Thipprakmas, M. Murakawa, J.C. Hung, Y.-C. Tsai, C.-H. Hung, Flexible Manufacturing System For Vibration Assisted Microforming, Simulation of ultrasonic-vibration drawing using the finite element method (FEM) 140 (2003) 30-35

[3] K. Siegert, A. Mock, Wire drawing with ultrasonically oscillating dies, J. Mater. Proc. Technol. 60 (1996) 657660

[4] K. Siegert, J. Ulmer, Superimposing Ultrasonic Waves on the Dies in Tube and Wire Drawing, J. Eng. Mater. Technol. 123 (2001) 517-523

[5] Y. Ashida, H. Aoyama, Press forming using ultrasonic vibration, J. Mater. Proc. Technol. 187 (2007) 118-122

[6] S.A.A. Akbari Mousavi, H. Feizi, R. Madoliat, Investigations on the effects of ultrasonic vibrations in the extrusion process, J. Mater. Proc. Technol. 187-188 (2007) 657-661

[7] M. Rosochowska, A. Rosochowski, FE simulation of ultrasonic back extrusion, ESAFORM Conference on Material Forming, 2007, pp. 564-569

[8] V.C. Kumar, I.M. Hutchings, Reduction of the sliding friction of metals by the application of longitudinal or transverse ultrasonic vibration, Tribol. Int. 37 (2004) 833-840

[9] M. Lucas, Y. Daud, A finite element model of ultrasonic extrusion, J. Phys. 181 (2009) 012-027

[10] K. Chen, N. Zhou, B. Liu, S. Wen, Effect of vibration extrusion on the structure and properties of high-density polyethylene pipes, Polym. Int. 58 (2009) 117-123

[11] S.A. Aziz, M. Lucas, The Effect of Ultrasonic Excitation in Metal Forming Tests, Appl. Mech. Mater. 24-25 (2010) 311-316

[12] M. Lucasa, Z. Huanga, M.J. Adams, Influence of ultrasonic upsetting of a model paste, Ultrasonics 40 (2002) $43-48$

[13] J.-C. Hung, C. Hung, The influence of ultrasonicvibration on hot upsetting of aluminium, Ultrasonic 43 (2000) 692-698

[14] Y. Daud, M. Lucas, Z. Huang, Modelling the effects of superimposed ultrasonic vibrations on tension and compression tests of aluminium, J. Mater. Proc. Technol. 186 (2007) 179-190
[15] Y. Daud, M. Lucas, Z. Huang, Superimposed ultrasonic oscillations in compression tests of aluminium, Ultrasonics 44 (2006) 511-515

[16] J.-C. Hung, Y.-C. Tsai, C. Hung, Frictional effect of ultrasonic-vibration on upsetting, Ultrasonics 46 (2007) $277-284$

[17] N.V. Polyakov, N.V. Mikhailov, A Study of vibrationassisted deformation of metals, Fiziko-Khimicheskaya Mekhanika Materialov 2 (1966) 482-484

[18] R. Ly, C. Giraud-Audine, G. Abba, R. Bigot, Experimentally validated approach for the simulation of the forging process using mechanical vibration, Int. J. Mater. Forming 2 (2009) 133-136

[19] R. Ly, C. Giraud-Audine, G. Abba, R. Bigot, Longitudinal vibrations modeling of a piezoelectric actuator used in forming process, Proceedings of the 2009 IEEE International Conference on Mechatronics, 2009

[20] A. Khan, Integration and Optimization of vibration in Metal Forming Process, Ph.D. thesis, ENSAM Metz, 2013

[21] K. Armaghan, C. Giraud-Audine, G. Abba, R. Bigot, Effects of Vibration on metal forming process: Analytical approach and finite element simulations, AIP Conf. Proc. 1315 (2011) 787-792

[22] T.H. Nguyen, C. Giraud-Audine, B. Lemaire-Semail, G. Abba, R. Bigot, Modelling of forging processes assisted by piezoelectric actuators: principles and experimental validation, IEEE Trans. Ind. Appl. 50 (2014) 244-252

[23] J. Zhi-hong, C. Gai-pin, Volume effect preparatory research of vibrational rotary forging, Proc. Mech. Automation Control Eng. (MACE) 24-25 (2010) 56215625

[24] J. Zhi-hong, C. Gai-pin, Surface effect preparatory research of vibrational rotary forging, Adv. Mater. Res. 154-155 (2011) 1513-1517

[25] M. Lucas, Z. Huang, M.J. Adams, Modeling wall boundary conditions in an elasto-viscoplastic material forming process, J. Mater. Proc. Technol. 107 (2000) 267-275

[26] J.C. Hung, Y.C. Tsai, C. Hung, Frictional effects of ultrasonic-vibration on upsetting, Ultrasonics 46 (2009) 277-284

[27] Y. Daud, M. Lucas, Z. Huang, Superimposed ultrasonic oscillations in compression tests of aluminium, Ultrasonics 44 (2006) 511-515

[28] Y. Daud, M. Lucas, Z. Huang, Modelling the effects of superimposed ultrasonic vibrations on tension and compression tests of aluminium, J. Mater. Proc. Technol. 186 (2007) 179-190 